\title{
Úlcera genital por Klebsiella granulomatis (Donovanosis) en una pareja heterosexual
}

\author{
Viany Gaviria-Sánchez ${ }^{a}$, Alvaro Monterrosa-Castro ${ }^{1}$, Eduardo Fernández-Daza. ${ }^{2}$ \\ ${ }^{1}$ Ginecólogo, Profesor Titular, Director Grupo de Investigación Salud de la Mujer, Facultad de Medicina, Universidad de \\ Cartagena. ${ }^{2}$ Patólogo Clínico, Profesor Titular, Facultad de Medicina, Universidad de Cartagena. Cartagena, Colombia.
}

\footnotetext{
a Alumna, Integrante del semillero FEM-SALUD del Grupo de Investigación Salud de la Mujer, Facultad de Medicina, Universidad de Cartagena. Cartagena, Colombia.
}

\section{RESUMEN}

Antecedentes: La Donovanosis o granuloma inguinal, es una enfermedad infecciosa, inflamatoria crónica, usualmente ulcerativa, preferentemente de localización ano-genital, trasmitida sexualmente y causada por la bacteria Klebsiella granulomatis. Se caracteriza por la presencia de los cuerpos de Donovan, en la microscopía de la secreción de las úlceras. El objetivo es presentar un caso de donovanosis en una pareja heterosexual, que consultaron por la presencia simultánea de lesiones en la vulva y en el pene. Caso clínico: pareja conformada por una mujer de 21 años de edad y su pareja masculina de 24 años, de elevado nivel socioeconómico, que observaron simultáneamente la aparición de una lesión indolora, ulcerada, de bordes elevados y fondo limpio, tanto en el labio mayor de la vulva como en el cuerpo del pene. Negaron la práctica de coito anal o promiscuidad. Se sospechó Donovanosis, por lo cual se realizó extendido citológico de la secreción de la lesión y se encontraron con la tinción de Giemsa, los cuerpos de Donovan en la lesión de la mujer. La muestra tomada al varón fue insuficiente para el estudio. Se realizó manejo de la pareja con doxiciclina. El varón tuvo completa mejoría, pero la mujer por presentar recidiva se le agregó azitromicina. A los cuatro meses del diagnóstico, ambos estaban asintomáticos y con una pequeña área de cicatriz. Conclusión: La donovanosis es un cuadro infeccioso que amerita diagnóstico y adecuado tratamiento, ya que tiene potenciales complicaciones genitales e incluso extragenitales, que son consideradas secundarias y pueden llevar a graves afectaciones para la salud.

\section{PALABRAS CLAVE: Granuloma inguinal, Donovanosis, infecciones por Klebsiella, infección de transmisión sexual}

\section{SUMMARY}

Background: The Donovanosis or granuloma inguinale is an infectious, chronic inflammatory and usually ulcerative disease, preferentially of anogenital location, that is sexually transmitted and caused by the bacteria Klebsiella granulomatis. It is characterized by the presence of the Donovan bodies in the microscopy of the secretion of the ulcers. The objective is to present a case of Donovanosis in a heterosexual couple, who consulted by the simultaneous presence of an injury in the vulva and penis. Case record: Couple of a 21 year-old woman and a 24 year-old man of high socioeconomic level that observed simultaneously the apparition of a painless and ulcerated injury of elevated edge and clear base, as much in the labia majora of the vulva as in the body of the penis. They denied the practice of anal coitus or promiscuity. The Donovanosis was suspected and the cytological study of the secretion of the injury was carried out. The Donovan bodies were found in the injury of the woman with the Giemsa stain. The sample of the man was insufficient for the study. The treatment of the couple was done with Doxycycline. The man had complete improvement but the woman presented recurrence for what Azithromycin was added to the treatment. To the four months of the diagnosis, both of them were asymptomatic and they had a small area of scar. 
Conclusion: The Donovanosis is an infectious disease that merits diagnosis and appropriate treatment due to it has potential genital and extragenital complications that are considered side effect and could carry to severe alterations for the health

\section{KEYWORDS: Granuloma inguinale, Donovanosis, Klebsiella infections, sexually transmitted infection}

\section{INTRODUCCIÓN}

La Donovanosis, también llamada granuloma inguinal o granuloma venéreo, es una enfermedad infecciosa, de transmisión sexual, inflamatoria, crónica y rara, localizada en el área ano-genital (1).

En 1882 la enfermedad fue descrita por McLeod en la India. Veinte años más tarde, Donovan en esa misma ciudad, describió al agente causal. Una década más adelante Aragao y Vianna propusieron el uso de tartrato de potasio antimonio, denominado también tártaro emético, considerado la primera medicación efectiva para el tratamiento de la enfermedad, aunque después fue abandonado su uso por los elevados riesgos tóxicos (2).

La Donovanosis es causada por la bacteria Klebsiella granulomatis, anteriormente conocida como Calymmatobacterium granulomatis, microorganismo gram negativo, intracelular, pleomórfico, aeróbico facultativo e inmóvil, que mide entre 1 a 2,5 $\mu \mathrm{ms}(1,3,4)$. Tiene un periodo de incubación entre una a doce semanas, aunque se ha señalado que puede llegar a ser hasta de varios meses (2). Goldbeer (5) en 1962 propuso que el hábitat natural de la bacteria era el intestino humano y las infecciones de la piel se sucedían directamente por coito anal o indirectamente por contaminación de los genitales con materia fecal.

La infección es más prevalente en niveles socioeconómicos bajos, es endémica en áreas rurales de regiones tropicales y subtropicales. Se encuentra más frecuentemente en Nueva Guinea, Sudáfrica, Brasil e India, así como entre aborígenes del norte de Australia y menos presente en los Estados Unidos de América $(6,7)$. Existen casos reportado en Centroamérica y el Caribe, mientras que en Perú es la primera causa de ulceras genitales crónica en pacientes con inmunodeficiencia $(2,8)$.

O’Farrell (8) ha señalado que el diagnóstico de la enfermedad suele no ser realizado en muchos lugares, con lo cual se subestima su real prevalencia. También es posible que los diagnósticos no se realicen debido a la carencia de experticia para el diagnóstico de la entidad o al uso indiscriminado de antibióticos (2). La incidencia entre sexos varía según los estudios y no se observa predilección por un género en particular. No se han reportado infecciones congénitas como resultado de infección fetal, y los casos en niños se asocian a contacto con adultos infectados, aunque no necesariamente por abuso sexual (9).

Se ha considerado que la epidemiologia e incluso la patogénesis de la entidad no están adecuadamente dilucidadas y se requiere más estudios al respecto (10). El objetivo es presentar un caso de Donovanosis en una pareja heterosexual que consultaron por la aparición de una lesión simultánea en vulva y pene.

\section{Caso clínico}

Pareja heterosexual conformada por una mujer de raza blanca de 21 años de edad y su compañero marital de raza negra de 24 años, profesionales del área de la salud, naturales y residentes en Cartagena de Indias, Colombia, sin visitas a otros países en los cinco años anteriores. Ambos pertenecían a estrato socioeconómico alto, estaban en buenas condiciones de salud y nutricional, no recibían medicamentos de forma regular, no tenían adicción al alcohol, tabaco o farmacodependencia. No poseían antecedentes de patológicos de interés, negaron practicar coitos anales e informaron no tener otras parejas sexuales.

La mujer presentó un nódulo eritematoso e indoloro que progresó a una lesión ligeramente ulcerada, de bordes elevados e hipertróficos y fondo limpio, en el labio mayor izquierdo de la vulva, el cual aumentó de tamaño de forma lenta, teniendo una semana de evolución al momento de la consulta (Figura 1). El compañero sexual también presentó de forma simultánea, la aparición de una lesión similar, de idéntico comportamiento, en el cuerpo del pene (Figura 2).

Fuera de las lesiones descritas, el examen clínico de ambas personas fue completamente normal. En la mujer se encontraron adenopatías inguinales bilaterales no dolorosas, al parecer inespecíficas y de curso mayor al que tenían las lesiones genitales. Las pruebas serológicas para sífilis y HIV fueron negativas en la pareja.

Con impresión clínica de úlceras genitales causadas por Donovanosis, se realizó frotis de la secreción, tomada con un hisopo, de las lesiones presentes en la vulva y pene. Los extendidos fueron coloreados con tinción de Giemsa para valoración 


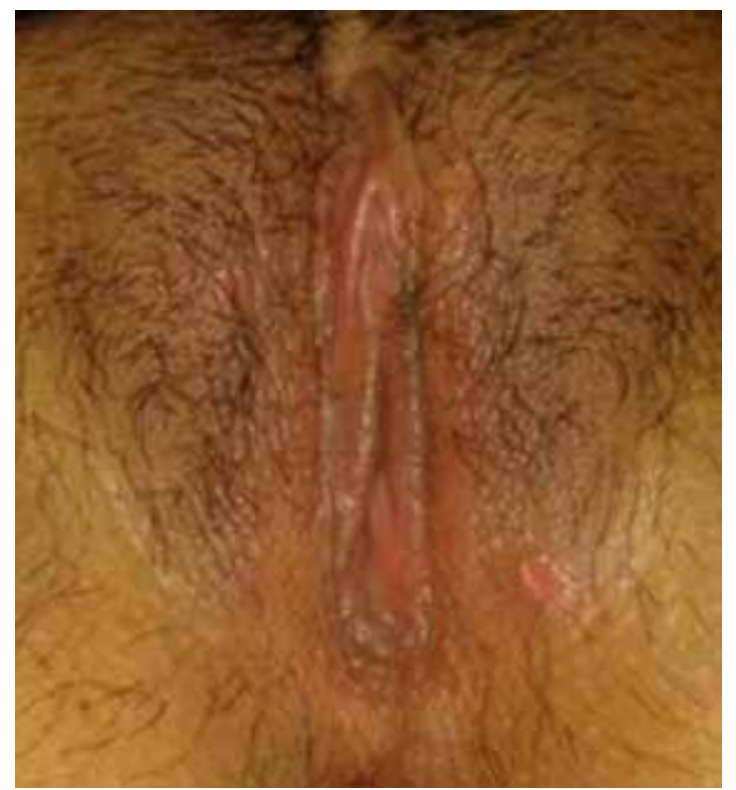

Figura 1. Genitales externos femeninos. Lesión ulcerada en labio mayor izquierdo de vulva, de bordes elevados e hipertróficos y fondo limpio.

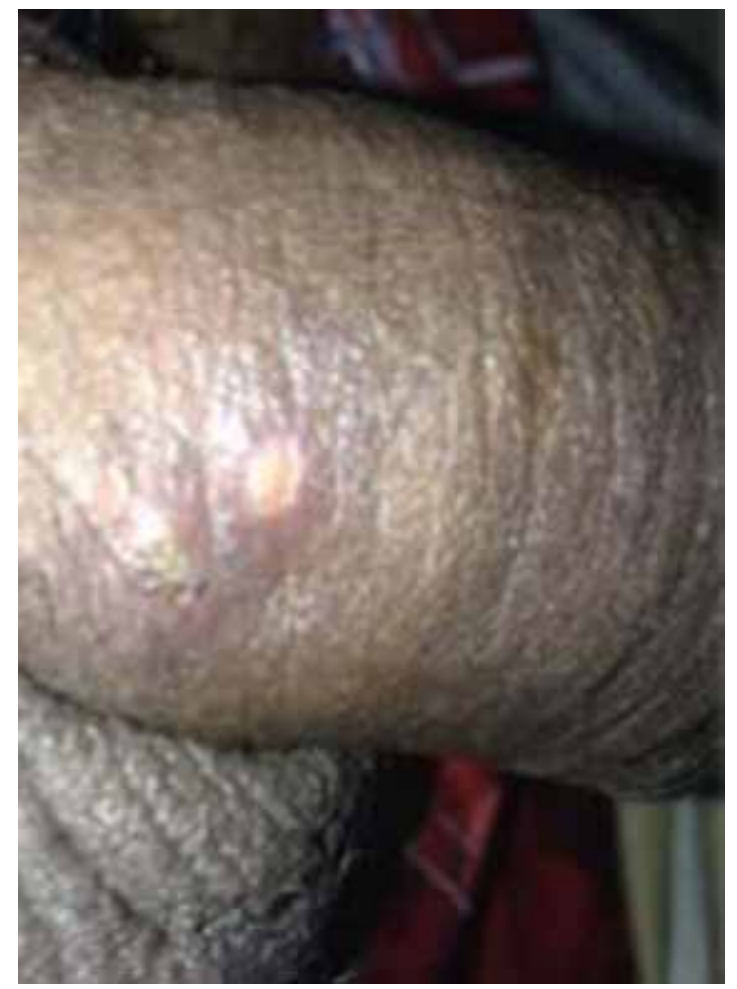

Figura 2. Genitales externos masculinos. Lesión ulcerada de bordes elevados e hipertróficos y fondo limpio en la cara izquierda del cuerpo del pene. microscópica. En la muestra de la mujer se observó la presencia de abundantes neutrófilos y células inflamatorias mononucleares, así como cocobacilos encapsulados diminutos en el interior de los macrófagos, que son denominados cuerpos de Donovan (Figura 3). Se realizó diagnóstico citológico de Donovanosis. La muestra obtenida de la úlcera genital del varón fue escasa y considerada insuficiente para el estudio. Tomando en consideración los hallazgos clínicos y de laboratorio se realizó diagnóstico de Donovanosis a ambos pacientes.

La pareja recibió tratamiento con doxiciclina, por vía oral, $100 \mathrm{mg}$ diarios por 4 semanas, con rápida mejoría. Dos semanas después de finalizada la terapéutica, la mujer presentó recidiva de la lesión, no así su compañero. A ella se le prescribió azitromicina vía oral, $500 \mathrm{mg}$ diarios por 21 días. Cuatro meses después del diagnóstico, ambos pacientes estaban asintomáticos y no había presentado nuevas lesiones ni crecimientos en el área previamente afectada, observándose solamente una pequeña área de hipocromía con aspecto de cicatriz (Figura 4).

\section{DISCUSIÓN}

El cuadro observado en ambos pacientes, corresponde a Donovanosis o granuloma inguinal, una importante causa de ulceración crónica en genitales. Es una infección transmitida sexualmente, que tiene como característica microscópica la presencia de cuerpos de Donovan. Es considerada una granulomatosis crónica, progresiva, que tiene asiento en el área genital, pudiendo comprometer piel como sucedió en los dos pacientes presentados, pero también las membranas mucosas (2). No se identificaron casos publicados de compromiso simultáneo en parejas sexuales.

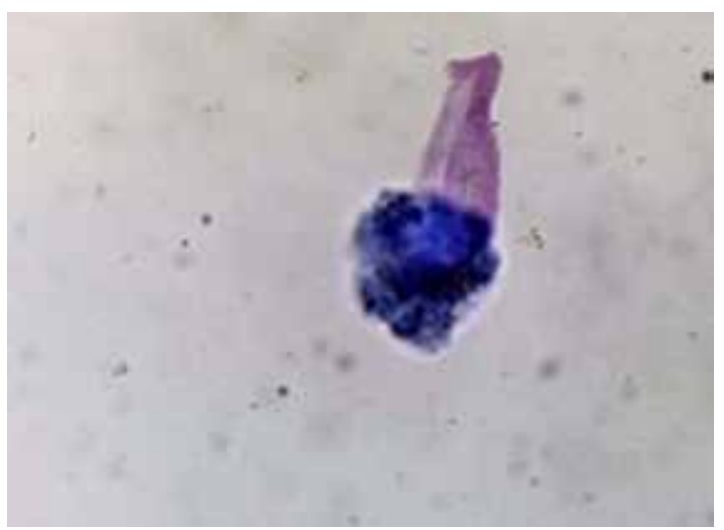

Figura 3. Cuerpos de Donovan en el frotis de la secreción de la lesión ulcerada del área genital femenina. Coloración de Giemsa. 40x. 

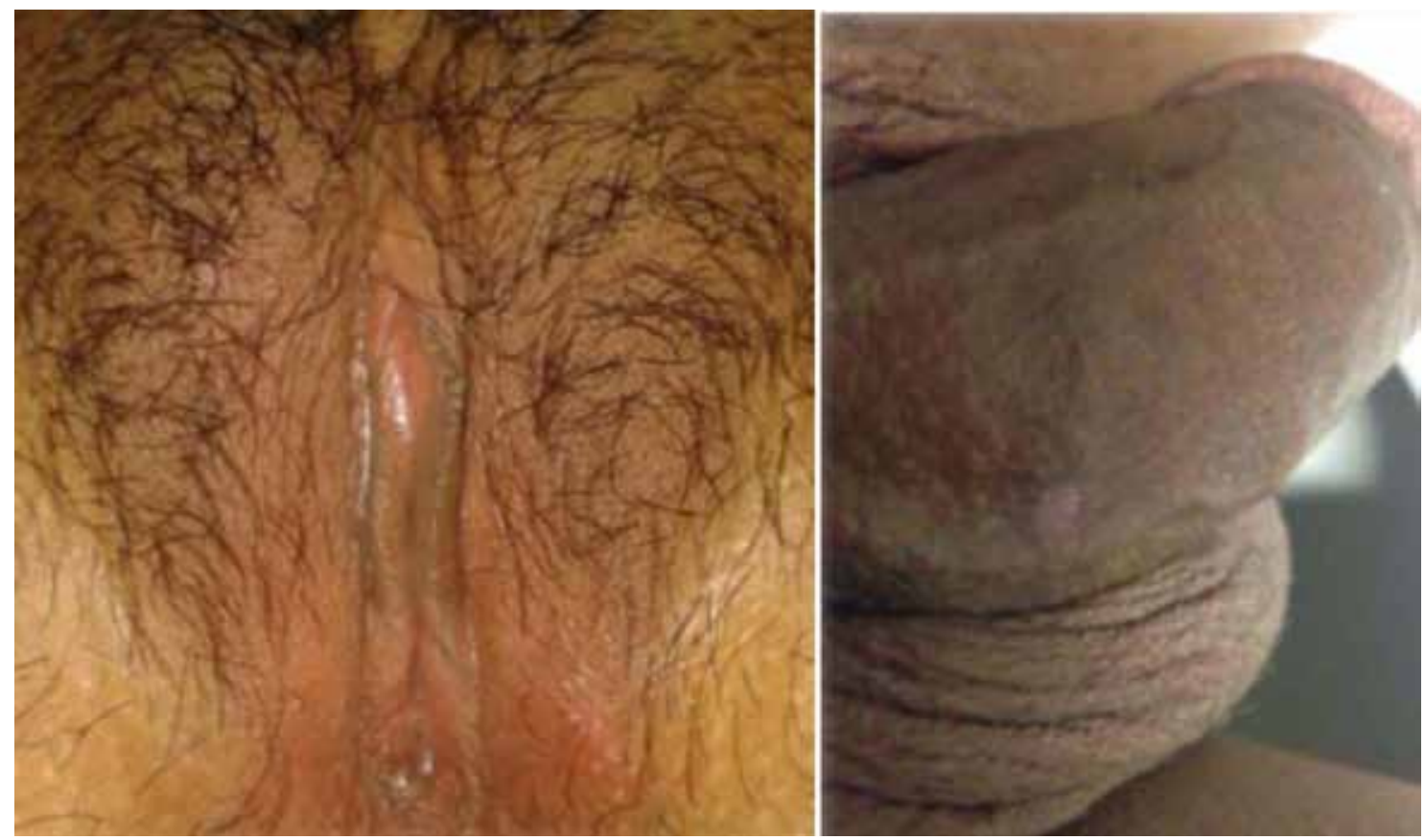

Figura 4. Genitales externos femenino y masculino. Dos meses posteriores al diagnóstico.

Clínicamente la Donovanosis es similar al linfogranuloma venéreo, chancro sifilítico primario y algunas formas secundarias, enfermedad de Ducrey o chancroide, úlceras herpéticas crónicas, condilomas acuminados ulcerados, infecciones micóticas o bacterianas y carcinoma espinocelular, debiendo realizarse siempre diagnóstico diferencial con todas ellas $(2,9,11)$.

Samuel y cols (12), han señalado la presencia concomitante de Donovanosis con sífilis y herpes simple. Las formas genitales de la Donovanosis se presentan en más del $90 \%$ de los casos (4) y también se deben diferenciar de otras lesiones no infecciosas poco comunes, como el síndrome de Behçet, erupción secundaria a medicamentos, psoriasis, trauma sexual y de la granulomatosis de Wegener (11).

Los pacientes presentados experimentaron la forma clínica más frecuente y característica de la enfermedad, lesiones eritematosas, ulcerativas superficiales, indoloras, con bordes elevados, fondo limpio y aspecto aterciopelado. La ubicación en los varones suele ser el pene, escroto, ingle o muslos, mientras que en las mujeres es la vulva, vagina y región perianal. Ocasionalmente pueden ser sangrantes. Cuando afectan el cuello uterino pueden tener la apariencia de un carcinoma cervical. Las úlceras son más frecuentes en hombre no circuncidados y en parejas con pobre higiene genital (2).
Se ha observado relación entre la presencia de Donovanosis y $\mathrm{VIH}$, aunque no se conoce si esta incrementa el riesgo de adquirir VIH (7). No obstante algunos autores señalan que la Donovanosis es potencialmente un importante factor de riesgo para la transmisión del VIH (4). La coexistencia de las dos infecciones, se expresan por úlceras persistentes y de periodos prolongados, que ameritan tratamientos más duraderos. El tamaño de las úlceras y la presentación clínica no suele ser diferente a la de los pacientes libres de VIH (9).

Se debe tener presente que la Donovanosis, además de ulcerativa, en la cual se encuentra abundante tejido granulomatoso en los bordes de la ulceración, también puede ser vegetativa o acompañarse de elefantiasis, un síndrome caracterizado por el aumento enorme de los órganos genitales externos. La forma elefantiásica ocurre a partir de formas ulcerativas crónicas, en las cuales se ha producido fibrosis dérmica extensa con deterioro de los linfáticos. La elefantiasis suele encontrarse más frecuentemente en mujeres y muy ocasionalmente en hombres, en ellas puede conllevar al establecimiento de fimosis genital con estenosis de la uretra, vagina y el ano (10).

O'Farrell y Moi (13), en 2010 propusieron que la Donovanosis genital tenía cuatro tipos de lesiones y las denominó: úlcero-granulomatosa (caso presentado), hipertrófica, necrótica y esclerótica o cicatricial. 
Se ha señalado que la Donovanosis puede adquirir curso de crecimiento agresivo durante el embarazo, con aumento en el número y recidivas de las lesiones. Si los genitales internos están afectados, se incrementa sensiblemente el riesgo de diseminación hematógena y se pueden presentar complicaciones para el embarazo y el parto. La operación cesárea se debe indicar cuando existe riesgo de laceraciones perineales (4). Aquellas mujeres que presenten lesiones atípicas o no diagnosticadas a nivel genital y experimenten crecimiento o modificaciones durante la gestación, se debe considerar la posibilidad de Donovanosis (8). La explicación para el cambio en el comportamiento biológico de la entidad es que los mecanismos de la inmunidad mediada por células, deprimidas en el embarazo, pueden favorecer las infecciones causadas por gérmenes intracelulares (4).

Como se observó en los pacientes presentados, suele haber ausencia de linfadenopatía en la región inguinal, es ligera la reacción inflamatoria local y no hay dolor. La enfermedad se propaga por continuidad y por mecanismo de auto-inoculación, en el caso presentado, la lesión femenina estaba en el labio mayor izquierdo y la masculina en la cara lateral izquierda del pene, en ambas personas las lesiones eran únicas. No obstante la lesión inicial puede dar origen a otras lesiones que aparecen de forma centrifuga y también frecuentemente en áreas de contacto. Es común observar la presencia de otras bacterias en el interior de la lesión (2), en los casos presentados no se realizaron búsqueda de ellas ni se practicaron cultivos de las secreciones obtenidas, las cuales eran escasas. La diseminación hematógena es rara, pero puede ocurrir cuando no se realiza tratamiento o la administración del mismo es insuficiente (6).

Si bien el diagnóstico se puede realizar con las características clínicas de la lesión, es importante la confirmación microscópica por medio del estudio del exudado de la úlcera, coloreado con la tinción de Giemsa o Wright, e incluso realizar en casos selectos, valoración histopatológica de la biopsia de la ulceración (1), sobre todo cuando el citodiagnóstico no permite confirmar la impresión clínica, lo cual es una situación poco frecuente. Los estudios histopatológicos tienen mayor importancia en presencia de lesiones extensas que involucran necrosis o esclerosis y es necesario descartar la posibilidad de lesiones malignas (2).

No se han establecido medios de cultivo adecuados y rutinariamente no están disponibles. La detección del microorganismo por medio de pruebas de detección de antígenos o fijación de complemento no tienen relevancia. Técnicas de detección por reacción en cadena de la polimerasa permiten la reclasificación del microorganismo, pero su uso debe estar restringido a programas de radicación de la enfermedad (7).

Se han descrito la presencia de lesiones extragenitales en la cavidad oral, faringe, laringe, abdomen, mucosa nasal e incluso huesos, especialmente en la tibia. Las lesiones de la cavidad oral son las más frecuentes de las lesiones extragenitales y al acompañarse de la caída de piezas dentarias, se debe buscar compromiso óseo maxilar por Donovanosis (14). También se puede presentar diseminación a la cavidad abdominal comprometiendo intestinos, bazo, hígado, útero y ovarios, ocurriendo estos casos severos, principalmente en áreas endémicas. Los pacientes presentaran fiebre, sudoración nocturna, anemia, pérdida de peso y estado tóxico, con riesgo de muerte si el diagnóstico y tratamiento no son realizados de manera temprana. Está demostrada la diseminación hematógena a huesos, articulaciones e hígado (8). Las lesiones extragenitales de la Donovanosis, son exclusivamente secundarias a las lesiones de los genitales o del ano y se presentan entre el 6 al $10 \%$ de los casos $(2,4)$. Las lesiones extragenitales se deben diferenciar de otras úlceras granulomatosas como la tuberculosis cutánea, paracoxidiomicosis, leishmaniasis, pioderma gangrenoso, rinoescleroderma y el granuloma medio facial (2).

Desde 1987, Jardim (15), propuso una clasificación para identificar las diferentes formas de presentación de la Donovanosis (Tabla I).

Tabla I

CLASIFICACIÓN DE LA DONOVANOSIS*

\begin{tabular}{ll}
\hline Ubicación de las lesiones & Tipos de lesiones \\
\hline & - Úlceras Con bordes hipertróficos \\
Genitales y perigenitales & - Úlcero-vegetativas bordes lisos \\
& - Vegetativas \\
& - Elefantiásicas
\end{tabular}

Extragenitales

$\left({ }^{\star}\right)$ Jardim ML. Donovanose: proposta de Clasificao Clinica. An Bras Dermatol 1987;62(3):169-72. 
Diferentes propuestas terapéuticas han sido señaladas en la última década, realizadas por la Organización Mundial de la Salud (OMS), Centro para la Prevención y Control de las Enfermedades (CDC) y el Ministerio de Salud del Brasil (MSB). La Tabla II presenta los medicamentos de primera línea y las alternativas que se han sugerido para el tratamiento de la Donovanosis. Todos los fármacos se administran por vía oral y al menos por tres semanas $(2,3)$.

Los pacientes afectados deben continuar bajo observación clínica hasta que desaparezcan completamente los signos y síntomas de la enfermedad. No existe consenso sobre cuál de los diferentes regímenes terapéuticos es el mejor. También puede ser útiles la minociclina, ceftriaxona, tianfenicol, trovofloxacina y norfloxacina $(9,16)$. Anteriormente se recomendaban múltiples dosis y varios medicamentos por periodos largos de tiempo, hoy día existe tendencia a administrarlos sin combinaciones y entre cuatro a seis semanas (7). Debido a que son raras las resoluciones espontáneas y la regla es la evolución progresiva y lenta; siempre debe realizarse tratamiento (4).

En el caso presentado se realizó tratamiento a los dos integrantes de la pareja ya que ambos presentaban lesiones y debieron pasarla rápidamente del uno al otro. Llamativamente, Costa y cols (17), consideran que la Donovanosis es una entidad de baja infectividad y usualmente no es necesario el tratamiento a la pareja sexual, no se identificaron otras posturas al respecto. No se pudo precisar cuál de las dos personas de la pareja presentada transmitió la enfermedad a la otra.

Finalmente y como se ha señalado, la Donovanosis es una enfermedad crónica, de transmisión sexual, de prevalencia incierta, con zonas endémicas y con posibles complicaciones severas y alto el porcentaje de subdiagnósticos. Tomando en consideración las condiciones actuales de vida que favorecen las migraciones, con fácil transitar hasta o desde zonas endémicas, es necesario fortalecer

Tabla II

TRATAMIENTO DE LA DONOVANOSIS

\begin{tabular}{|c|c|c|c|}
\hline & OMS (2003) & CDC (2010) & MSB (2006) \\
\hline $\begin{array}{l}\text { Primera línea } \\
\text { terapéutica }\end{array}$ & $\begin{array}{c}\text { Azitromicina } \\
1 \text { gramo el primer día y } \\
\text { continuar con } 500 \mathrm{mg} \\
\text { diarios } \\
\text { o } \\
\text { Doxiciclina } \\
100 \text { mg dos veces al día }\end{array}$ & $\begin{array}{c}\text { Doxiciclina } \\
100 \text { mg dos veces al día }\end{array}$ & $\begin{array}{c}\text { Doxiciclina } \\
100 \text { mg dos veces al día }\end{array}$ \\
\hline
\end{tabular}

\begin{tabular}{|c|c|c|c|}
\hline $\begin{array}{l}\text { Segunda línea } \\
\text { terapéutica }\end{array}$ & $\begin{array}{c}\text { Eritromicina } \\
500 \text { mg cuatro veces al día } \\
\text { o } \\
\text { Tetraciclina } \\
500 \text { mg cuatro veces al día } \\
\text { o } \\
\text { Trimetoprim-Sulfametoxa- } \\
\text { zol } 400 \mathrm{mg} / 80 \mathrm{mg} \\
\text { dos veces al día } \\
\text { por } 14 \text { días }\end{array}$ & $\begin{array}{c}\text { Azitromicina } \\
1 \text { gramo por una semana } \\
\text { o } \\
\text { Ciprofloxacina } \\
750 \text { mg dos veces al día } \\
\text { o } \\
\text { Eritromicina } \\
500 \text { mg cuatro veces al día } \\
\text { o } \\
\text { Trimetoprim-Sulfametoxa- } \\
\text { zol } 400 \mathrm{mg} / 80 \mathrm{mg} \\
\text { dos veces al día }\end{array}$ & $\begin{array}{c}\text { Eritromicina } \\
500 \mathrm{mg} \text { cuatro veces al día } \\
\text { o } \\
\text { Trimetoprim-Sulfametoxa- } \\
\text { zol } \\
400 \mathrm{mg} / 80 \mathrm{mg} \\
\text { dos veces al día } \\
\text { o } \\
\text { Tetraciclina } \\
500 \mathrm{mg} \text { cuatro veces al día } \\
\text { o } \\
\text { Azitromicina } \\
1 \text { gramo el primer día y } \\
\text { continuar con } 500 \mathrm{mg} \\
\text { diarios }\end{array}$ \\
\hline
\end{tabular}

Eritromicina

500 mg cuatro veces al día

al menos tres semanas y

hasta la cicatrización

completa de las lesiones
Eritromicina

$500 \mathrm{mg}$ cuatro veces al día al menos tres semanas y hasta la cicatrización completa de las lesiones 
el conocimiento sobre Donovanosis entre los profesionales de atención básica y especializada en salud, para identificar casos importados desde otros países, sin olvidar la existencia de cuadros nativos, como el presentado

\section{CONCLUSIÓN}

La Donovanosis es una infección de transmisión sexual, que puede ser un desafío diagnóstico, por las diferentes formas de presentación genital y extragenital. La Donovanosis es parte de las lesiones ulcerosas de los genitales. Tiene buen pronóstico, aunque se han observado dos escenarios clínicos de mayor riesgo: el embarazo y el $\mathrm{VIH}$.

\section{REFERENCIAS}

1. Richens J. The diagnosis and treatment of Donovanosis (granuloma inguinale). Genitourin Med 1991;67(6):441-52.

2. Ferreira $P$, Macedo E, Belda W. Donovanosis. BJID 2008;12(6):521-5.

3. Centers for Disease Control and Prevention (CDC). Sexually transmitted diseases treatment guidelines. 2010. MMWR 2010;59:RR-12. Disponible en: http:// www.cdc.gov/mmwr/pdf/rr/rr5912.pdf. Acceso: 1 de Mayo de 2015.

4. Liverani CA, Lattuada D, Mangano S, Pignatari, Puglia D, Monti E, et al. Hypertrophic Donovanosis in a young pregnant woman. J Pediatr Adolesc Gynecol 2012;25(4):e81-e83.

5. Goldber J. Studies on granuloma inguinale. Isolation of bacterium from faeces of a patient with granuloma inguinale. Br J Vener Dis 1962;38(2):99-102.

6. Sociedad Argentina de Infectología. Recomendaciones para el seguimiento y tratamiento de las enfermedades de transmisión sexual. 2010. Disponible en: https://es.scribd.com/doc/133648925/ Recomendaciones-ETS-SADI-2010. Acceso el 1 de Mayo de 2015.

7. Bowden F. Donovanosis in Australia: going, going. Sex Transm Infect 2005;81(5):365-6.

8. O'Farrell N. Donovanosis: an Update. Int J STD AIDS 2001;12(7):423-7.

9. O'Farrell N. Donovanosis. Sex Trans Infect. 2002;78(6):452-7.

10. Veeranna S, Raghu TY. A clinical and investigational study of donovanosis. Indian J Dermatol Venereol Leprol 2003;69(2):159-62.

11. Roett M, Mayor M, Uduhiri K. Diagnosis and management of genital ulcers. Am Fam Physician 2012;85(3):254-62.

12. Sammuel M. Aderogba K. Dutt N, et al. A hat trick ulcerating pathogens in a single genital lesion. Int $\mathrm{J}$ STD AIDS 2007;18(1):65-6.

13. O Farrell N. Moi H; IUSTI/WHO European STD guidelines Editorial Board. European guideline for the management of donovanosis. 2010. Int J STD AIDS 2010;21(9):609-610.

14. Veerenna S, Raghu TY. Oral Donovanosis. Int J STD AIDS 2002;13(12):855-6.

15. Jardim ML. Donovanose: proposta de Clasificao Clinica. An Bras Dermatol 1987;62(3):169-72.

16. The Infectious Diseases Society of Taiwan. Taiwan AIDS Society, Taiwan Urological Association. Taiwan Association of Obstetrics and Gynecology. Medical Foundation in Memory of Dr. Deh-Lin Cheng. Foundation of Professor Wei-Chuan Hsieh for Infectious Diseases Research and Education. CY Lee's Research Foundation for Pediatric Infectious Diseases and Vaccines. Guideline on antimicrobial therapy of sexually transmitted diseases in Taiwan. J Microbiol Immunol Infect 2010;43(1):81-3.

17. Costa MC, Bornhausen Demarch E, Azulay DR, Périssé AR, Dias MF, Nery JA. Sexually transmitted diseases during pregnancy: a synthesis of particularities. An Bras Dermatol. 2010;85(6):767-85. 